\title{
On the Analytic Curve of $C^{2}$ which is not Omitted by Every Fatou-Bieberbach Domain
}

\author{
YUKINOBU ADACHI* \\ Yukinobu Adachi, Kurakuen, Nishinomiya, Hyogo, Japan \\ *Corresponding author: fwjh5864@nifty.com \\ Received December 17, 2014; Revised January 27, 2015; Accepted February 06, 2015
}

\begin{abstract}
Let $C$ be an irreducible (may be transendental) analytic curve whose genus is geater than 1 . Then every Fatou-Bieberbach domain does not omit $C$.

Keywords: fatou-bieberbach domain, hyperbolic cuve, transcendental algebraic type curve, kobayashi hyperbolic

Cite This Article: YUKINOBU ADACHI, "On the Analytic Curve of $\mathrm{C}^{2}$ which is not Omitted by Every Fatou-Bieberbach Domain.” American Journal of Mathematical Analysis, vol. 3, no. 1 (2015): 19-20. doi: 10.12691/ajma-3-1-4.
\end{abstract}

\section{Introduction}

We call that a domain $\Omega \subsetneq \mathrm{C}^{2}$ is a Fatou-Bieberbach domain if $\Omega$ is biholomorphic to $C^{2}$.

We call that an irreducible algebraic curve $A$ of $C^{2}$ is hyperbolic when $g(A)>0$ or else $g(A)=0$ and the set $A \cap L_{\infty}$ consists more than two different points, where $g(A)$ is the genus of $A$ and $L_{\infty}$ is the line at infinity.

In the previous paper [2], we proved the case $A$ (theorem 2.1). In this paper, every Fatou-Bieberbach domain does not omit $C$ which is the same in Abstract (Theorem 3.12). Moreover we prove that the property that an analytic curve of $\mathbf{C}^{2}$ does not omit every FatouBieberbach domain is unchanged one by a transformation of $\operatorname{Aut}\left(\mathbf{C}^{2}\right)$ (Theorem 3.7). Therefore every FatouBieberbach domain does not omit $T(A)$ with $T \in \operatorname{Aut}\left(\mathbf{C}^{2}\right)$. And we prove also that there is an algebraic type curve of $\mathbf{C}^{2}$ which is isomorphic to an algebraic curve of any topological type such that it can not be transformed to an algebraic curve by any $T \in \operatorname{Aut}\left(\mathbf{C}^{2}\right)$ (Proposition 3.3).

\section{Preliminary}

Theorem 2.1 (Theorem 2.1 in [2]). Let $A$ be $a$ hyperbolic algebraic curve of $\boldsymbol{C}^{2}$. Then every FatouBieberbach domain $\Omega$ does not omit $A$.

Remark 2.2 (Theorem 2.2 in [2]). Let $\Omega_{0}$ be every Fatou-Bieberbach domain in $\mathbf{C}^{2}$ arising from a polynomial automorphism, namely a polynomial basin. Then $\Omega_{0}$ does not omit every algebraic curve of $\mathbf{C}^{2}$.

Theorem 2.3 (Theorem 4.1 in Buzzard and Fornaess [4]). Let $X$ be an arbitrary analytic curve of $C^{2}$. Then there is a Fatou-Bieberbach domain $\Omega$ with $\Omega \supset X$ and $\Omega \neq C^{2}$ where some $\Phi: \Omega \rightarrow C^{2}$ is biholomorphic such that every nonconstant holomorphic map $f: C \rightarrow C^{2}$ intersects with $\Phi(X)$ at in_nite points and $C^{2}-\Phi(X)$ is Kobayashi hyperbolic.

Example 2.4 (Example 9.7 in [6]). There is a FatouBieberbach domain $\Omega 0$ such that $\bar{\Omega}_{0}$ namely the closure of $\Omega_{0}$ omits an algebraic complex line $L$.

Proposition 2.5 (Proposition 3.1 in [2]). For some transendental complex line $\tilde{L}$ such that there is a FatouBieberbach domain $\Omega 0$ with $\bar{\Omega}_{0} \cap \tilde{L}=\emptyset$.

From Theorem 2.3, Example 2.4 and Proposition 2.5, following theorem is easy to see.

Theorem 2.6. Let $C$ be an analytic curve of $C^{2}$. Then every Fatou-Bieberbach domain does not omit $C$ or some (not all) Fatou-Bieberbach do-main $\Omega_{0}$ omits $C$.

\section{Conclusion}

Proposition 3.1. Let $\tilde{A}$ be a transendental hyperbolic curve of $\mathrm{C} 2$ which is isomorphic to a hyperbolic algebraic curve A. If $\tilde{A}$ is transformed to an alge-braic hyperbolic curve $A$ of $C^{2}$ by $T \in \operatorname{Aut}\left(C^{2}\right)$, then every FatouBieberbach domain does not omit $\tilde{A}$.

Proof. We assume that there is a Fatou-Bieberbach domain $\Omega$ with $\Omega \cap \tilde{A}=\emptyset$. Then $T(\Omega) \cap A=\emptyset$. and $T(\Omega)$ is biholomorphic to $\mathbf{C}^{2}$ by $T \circ \Phi$, where $\Phi$ is a biholomorphic map of $\Omega$ to $\mathbf{C}^{2}$. Therefore $T(\Omega)$ is a FatouBieberbach domain and it contradicts to Theorem 2.1.

Proposition 3.2. Let $X$ and $\Omega$ be the same of Theorem 2.3. Then $\Phi(X)$ can not be transformed to any algebraic curve $A$ which is hyperbolic or non by any $T \in \operatorname{Aut}\left(C^{2}\right)$.

Proof. We assume that $\Phi(X)$ is transformed to an algebraic curve $A$ by some $T \in \operatorname{Aut}\left(\mathbf{C}^{2}\right)$. As $T(\Omega)$ is a Fatou-bieberbach domain with $T \circ \Phi(X)=A$. Let $f: \mathbf{C} \rightarrow \mathbf{C}^{2}$ be a map to an algebraic complex line. Then $(T \circ \Phi(X)) \cap f(\mathbf{C})$ is a finite set of points at most and $\Phi(X) \cap(T-1 \circ f(\mathbf{C}))$ is also. It is a contradiction to Theorem 2.3. 
Proposition 3.3 (cf. Proposition 3.12 in [2]). There is a transcendental analytic curve of $\mathbf{C}^{2}$ which is isomorphic to an algebraic curve of any topo-logical type, that is an algebraic type curve of any type, such that it can not be transformed to an algebraic curve by any $T \in \operatorname{Aut}\left(\mathrm{C}^{2}\right)$. If we take $X$ an algebraic type curve of any topological type, $\Phi(X)$ which is the same notation of Theorem 2.3 is such one.

Proposition 3.4. Let $X, \Omega$ and $\Phi$ be the same of Theorem 2.3. Then every Fatou-Bieberbach domain $\Omega^{\prime}$ does not omit $\Phi(X)$.

Proof. If some $\Omega^{\prime}$ omits $\Phi(X), \Phi^{r^{-1}}: \mathbf{C}^{2} \rightarrow \Omega^{\prime} \subset \mathbf{C} 2-\Phi(X)$, where $\Phi^{\prime}$ is a biholomorphic map of $\Omega^{\prime}$ to $\mathbf{C}^{2}$. Since $\mathrm{C}^{2}-\Phi(X)$ is Kobayashi hyperbolic, $\Phi^{{ }^{-1}}$ is a constant map. It is a contradiction.

Proposition 3.5. Let $C$ be an analytic curve of $C^{2}$ which is transformed by $T \in \operatorname{Aut}\left(\mathrm{C}^{2}\right)$ to some analytic curve $C^{\prime}$ of $\boldsymbol{C}^{2}$ which does not omit every Fatou-Bieberbach domain. Then every Fatou-Bieberbach domain does not omit $C$.

Proof. We assume that there is a Fatou-Bieberbach domain $\Omega_{0}$ with $\Omega_{0} \cap \mathrm{C}=\emptyset$. Then $\mathrm{T}\left(\Omega_{0}\right) \cap \mathrm{T}(\mathrm{C})=\emptyset$. Since $\mathrm{T}\left(\Omega_{0}\right)$ is a Fatou-Bieberbach domain which omits $\mathrm{C}^{\prime}$ $=\mathrm{T}(\mathrm{C})$. It contradicts to the property of $\mathrm{C}$.

Corollary 3.6. Let $C$ be an analytic curve of $C^{2}$ which does not omit every Fatou-Bieberbach domain. Then $T(C)$ with every $T \in \operatorname{Aut}\left(\boldsymbol{C}^{2}\right)$ does not omits every FatouBieberbach domain.

Proof. Since $\mathrm{T}^{-1} \circ \mathrm{T}(\mathrm{C})=\mathrm{C}, \mathrm{T}(\mathrm{C})$ does not omit every Fatou-Bieberbach domain also by Proposition 3.5.

From Proposition 3.5 and Corollary 3.6 it is easy to see the following theorem.

Theorem 3.7. The property that the analytic curve of $\boldsymbol{C}^{2}$ does not omit every Fatou-Bieberbach domain is unchanged one by a transformation of $\operatorname{Aut}\left(\mathrm{C}^{2}\right)$.

From Proposition 3.4 and Theorem 3.7 it is easy to see the following corollary.

Corollary 3.8. Let $C$ be an analytic curve of $C^{2}$ which is transformed by some $T \in \operatorname{Aut}\left(\boldsymbol{C}^{2}\right)$ to $\Phi(X)$ where $X, \Omega$ and $\Phi$ are the same of Proposition 3.4. Then every FatouBieberbach domain $\Omega^{\prime}$ does not omit $C$.

Problem 3.9. Is there $\tilde{A}$ which is not transformed to an algebraic hyperbolic curve $A$ of $\mathbf{C}^{2}$ or $\Phi(X)$, which is the same notation of Theorem 2.3, by some $T \in \operatorname{Aut}\left(\mathbf{C}^{2}\right)$ ?

Proposition 3.10. Let $C$ be an irreducible analytic curve of $C^{2}$ with $g(C)>1$. Then $D=C^{2}-C$ is not biholomorphic to $C^{2}$, that is, $D$ is not a Fatou-Bieberbach domain.

Proof. We assume that there is a biholomorphic map $\Phi$ of $D$ to $\mathbf{C}^{2}(x, y)$ such as $\Phi: x=\xi(z, w), y=\eta(z, w)$ where $(z, w) \in D$. Let $P(x, y)$ be a nonconstant primitive polynomial, that is all level curve of $\{P(x, y)=\alpha\}$ is irreducible except at most finite number of $\alpha 1, \cdots, \alpha$. Then $P(\xi(z, w), \eta(z, w)) \in \mathcal{O}(D)$. If $C$ is an essential singular curve of $P(\xi, \eta)$, every level curve of $P(\xi, \eta)$ can not be analytically continued to every point of $C$ except at most one value $\alpha 0$ by well known Thullen's theorem.
Since $\left\{P(\xi, \eta)=\alpha, \alpha \neq \alpha_{0}, \alpha_{1}, \cdots, \alpha_{n}\right\}$ is considered as a Riemann surface $\mathrm{R}_{0}=\mathrm{R}-\left\{\mathrm{p}_{1}, \cdots, \mathrm{p}_{\mathrm{m}}\right\}$ where $\mathrm{R}$ is a compact Riemann surface, that is an algebraic type Riemann surface such as $\pi: \mathrm{R}_{0} \rightarrow\{\mathrm{P}(\xi, \eta)=\alpha\} \subset \mathbf{C}^{2}$ is the normalization. And at some $\mathrm{p}_{\mathrm{i}} \in\left\{\mathrm{p}_{1}, \cdots, \mathrm{p}_{\mathrm{m}}\right\}$ the cluster set of $\pi$ in $\mathbf{C}^{2}$ is $C$. Because it is a pseudoconcave set of $\mathbf{C}^{2}$ by Theorem 3.4 in [1] and it is a degeneration locus of Kobayashi pseudodistance by Theorem 3.6 in [1]. It is a contradiction to $\mathrm{g}(\mathrm{C})>1$ by Theorem 4 in [3].

Therefore $C$ is not an essential singular curve of $P(\xi, \eta)$, that is, $P(\xi, \eta)$ is at most meromorphically continued to $C$. Since $P(x, y) \in \mathcal{O}\left(\mathbf{C}^{2}\right), P(\xi, \eta)$ is holomorphically continued to $C$. We set such function as $F(z, w)$.

Since every level curve of $F(z, w)$ is holomorphically isomorphic to algebraic type Riemann surface and an analytic cuve of $\mathbf{C}^{2}, F(z, w)$ is an algebraic type entire function of Nishino's sence [5], namely in the class $(A)$. Then by principal theorem of [5], $F=\varphi \circ Q \circ T$ where $\varphi$ is a polynomial function of one complex variable because $P(x, y)$ is primitive, $Q$ is a primitive polynomial and $T \in$ $\operatorname{Aut}\left(\mathbf{C}^{2}\right)$.

Then $T_{\mid D}=\Phi$. It contradicts to the assumption. Then $D$ is not biholomorphic to $\mathbf{C}^{2}$.

Proposition 3.11. Let $C$ be an irreducible analytic curve of $C^{2}$ such that $D=C^{2}-C$ is not a Fatou-Bieberbach domain. Then every subdomain of $D^{\prime}$ of $D$ is not a FatouBieberbach domain.

Proof. If $D^{\prime}$ is a Fatou-Bieberbach domain, there is a biholomorphic map $\Phi$ of $\mathbf{C}^{2}$ to $\mathrm{D}^{\prime}$. Let I be an inclusion map of $D^{\prime}$ to $\mathrm{D}$ and $\{\mathrm{f}=0\}=\mathrm{C}$ where $\mathrm{f} \in \mathcal{O}\left(\mathbf{C}^{2}\right)$. Since $\mathrm{g}$ $=\mathrm{f} \circ \mathrm{I} \circ \Phi$ is an entire function of $\mathbf{C}^{2}$ and $\mathrm{g} \neq 0$. There is a complex line $\mathrm{L}$ of $\mathbf{C}^{2}$ and $\left.\mathrm{g}\right|_{\mathrm{L}}$ is considered as a transendental entire function of $\mathbf{C}$, it is a contradiction by little Picard theorem because $\left.\mathrm{I} \circ \Phi\right|_{\mathrm{L}}$ is an one to one map.

From Proposition 3.10 and 11, following theorem is easy to see.

Theorem 3.12. Let $C$ be the same of Proposition 3.10. Then every Fatou-Bieberbach domain does not omit $C$.

\section{References}

[1] Y. Adachi, A generalization of the big Picard theorem, Kodai Math. J., 18(1995), 408-424.

[2] Y. Adachi, Remarks about Fatou-Bieberbach domains and algebraic or non-algebraic curves in $\mathbf{C}^{2}$, Far East J. Math. Soc. (FJMS), 34 (2009), 369-376.

[3] Y. Adachi and Masakazu Suzuki, Degeneracy points of the Kobayashi pseudodistances on complex manifolds, Proceedings of Symposia in Pure Math., 52 (1991), Part 2, 41-51.

[4] G. T. Buzzard and J. E. Fornaess, An embedding of $\mathbf{C}$ in $\mathbf{C}^{2}$ with hyperbolic complement, Math. Ann. 306 (1996), 539-546.

[5] T. Nishino, Nouvelles recherches sur les fonctions entières de plusieurs variables conplexes (V) Fonctions qui se réduisent aux polynômes, J. Math. Kyoto Univ., 15 (1975), 527-553.

[6] J. P. Rosay and W. Rudin, Holomorphic maps from $\mathbf{C n}$ to $\mathbf{C n}$, Trans. A. M. S., 310 (1988), 47-86. 